\title{
Natural protection against harmful oxidation
}

Michelle JONES

Technical Manager Europe, ADM Natural Health and Nutrition

Church Manorway

Erith

Kent

DA8 1DL, UK

\begin{abstract}
Although still not conclusive, the studies described indicate that topically applied vitamin $E$ can provide protection against ultraviolet radiation when applied alone or in combination with other antioxidants. The general consensus is that, although less stable than the tocopherol esters when used in cosmetic systems, the alcohol form of alpha-tocopherol is the most effective topically-applied skin protectant. Increased UV protection potentially reduces the risk of skin cancer. However, the debate continues regarding the skin's ability to hydrolyse ester forms of alpha-tocopherol, which are used commercially because of their greater stability during processing and over time in these products. Further research is therefore needed in order to provide more definitive data on what potential benefits or possible disadvantages may be associated with use of the ester forms in these topical applications.
\end{abstract}

Key words: natural protection, harmful oxidation

secreting glands provide skin with some protection against harmful bacteria). These results suggested that vitamin $\mathrm{E}$ is supplied to the skin via sebaceous glands. This observation was further supported by the finding of a positive correlation between the levels of vitamin $\mathrm{E}$ and squalene in skin surface lipids (squalene is a marker of sebum secretion). Sebaceous gland secretion was found to be an effective means of transferring dietary vitamin $\mathrm{E}$ to skin where it may help protect both skin surface lipids and the upper stratum corneum (the outermost layer of the skin) from harmful oxidation. an aid to protecting the skin from externa damage caused by harmful ultraviolet rays, environmental pollutants and oxidative stresses, when topically applied. Consequently, manufacturers of cosmetics are now realising the potential of vitamin $E$ and increasingly incorporating it into skincare products such as moisturisers and sunscreens.

Here, we examine the natural skin protecting properties of vitamin $\mathrm{E}$, its depletion in dermal layers and benefits of supplementation. We also cite key studies into the topical application of vitamin $\mathrm{E}$ and the impact on future product development.

\section{Protecting skin naturally}

The skin is the body's first line of defence against the effects of atmospheric contaminants such as cigarette smoke and ultraviolet radiation. Although vitamin $\mathrm{E}$ is naturally present in the skin, studies have shown that it is susceptible to ultraviolet-induced depletion [2]. Levels of vitamin $E$ are at their lowest near to the surface of the skin, and increase towards the deeper layers. Researchers at the University of Jena have proposed a mechanism for the supply of vitamin E to the skin [3].

Researchers found that parts of the body with the highest concentrations of vitamin $\mathrm{E}$ also had a higher sebaceous gland density (sebum-

\section{Vitamin E explained}

Four forms of vitamin $\mathrm{E}$ are present in nature: alpha-, beta-, gamma- and delta-, and although gamma is the most abundant naturally-occurring form of tocopherol in the human food supply chain, it is the alpha form, d-alpha-tocopherol or RRR-alpha-tocopherol, that is retained primarily by the human body. Natural-source d-alpha-tocopherol is one stereoisomer whereas the synthetic "dl-alphatocopherol" is a combination of eight stereoisomers, only one of which is identical to $\mathrm{d}$-alpha-tocopherol. The remaining seven stereoisomers have different stereochemistries, with resultant lower biological activity relative to the RRR isomer.

Tocotrienols are closely related to the tocopherols and are also present in some oil-bearing crops at low levels. Tocotrienols differ from tocopherols by containing three double bonds in the phytyl side chain. These double bonds give the tocotrienols fluidity, enabling the body to incorporate them into cell membranes with ease. Compared with tocopherols, it has been proposed that tocotrienols may provide additional benefits [4].

\section{Tocopherols versus tocotreniols}

Alpha-tocopherol is the most abundant form of vitamin $\mathrm{E}$ naturally present in the skin, although there are also small amounts of gamma-tocopherol, as well as alpha- and gamma-tocotrienol. In research demonstrating the presence of alpha- and gamma-tocotrienol in the skin, it has also been proposed that tocotrienols may offer skin barrier protection and growth-modulating properties [4], in addition to antioxidant activity and photoprotection.

In a study at the department of dermatology at the Goethe University in Frankfurt, the antioxidant capabilities of tocopherols and tocotrienols in the epidermis were compared using a free radical scavenging technique [4]. Using this technique, no significant free-radical scavenging was observed for either alpha- or gamma-tocotrienol, whereas significant freeradical scavenging was demonstrated for both alpha- and gamma-tocopherol.

Data from this study along with observed levels in human skin suggest that alpha-tocopherol is quantitatively the most important form of vitamin $\mathrm{E}$ for protection against free radical attack. This is further emphasised by the comparison of levels of alpha-tocopherol and gammatocopherol depletion after skin was subjected to intense UV exposure: alpha-tocopherol was depleted by 0.75 MED (Minimal Erythema Dose) whereas gamma-tocopherol was depleted only after a very high dose [2] (figure 1).

\section{Vitamin E in sun protection products}

Vitamin $\mathrm{E}$ is available commercially as both the free alcohol and in esterified forms; both forms are used in topically applied skin protection products, but which is best? 


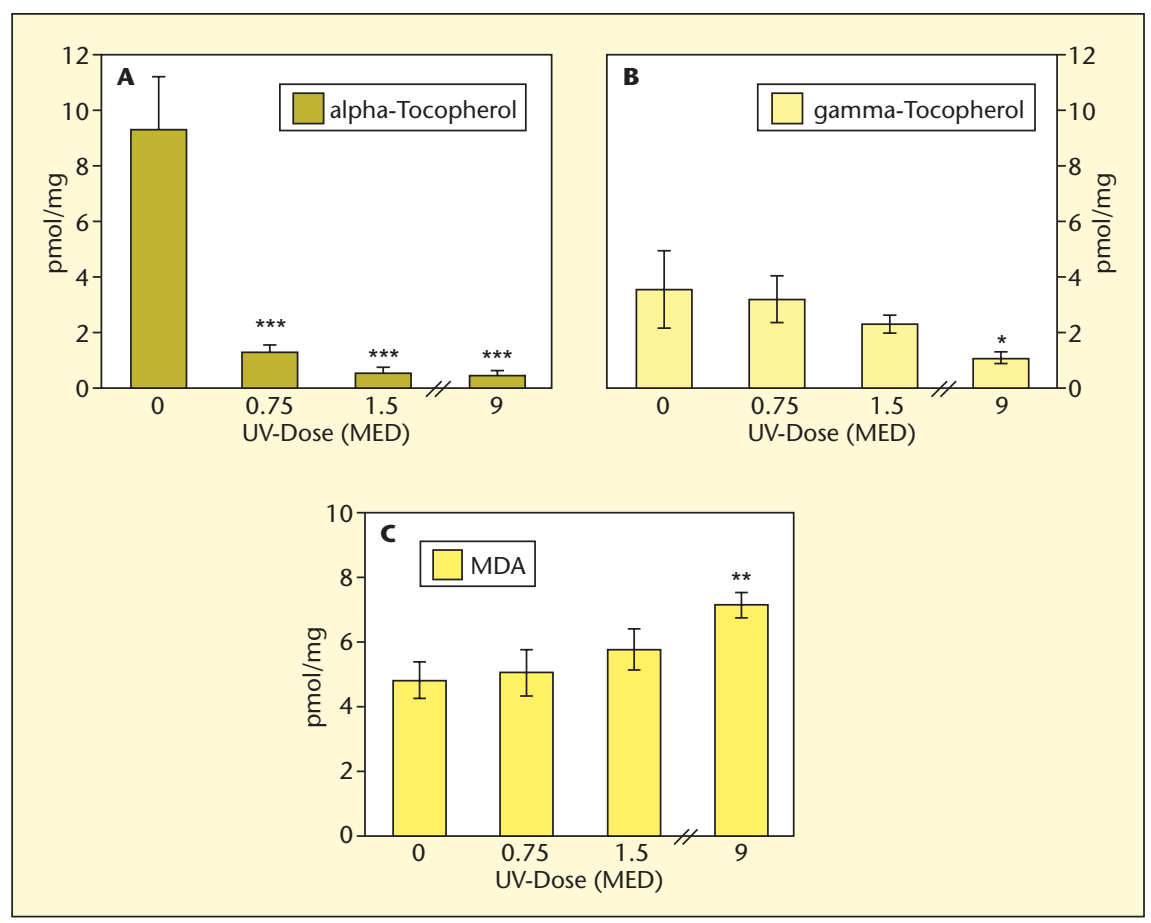

Figure 1. Tocopherols. Effect of UVR.

Animal studies have demonstrated that topical application of the free tocopherol form may prevent skin cancer and also inhibit ultraviolet (UV) B-induced immune susceptibility [6]. Subsequent studies in both animal and human skin models have compared the effects of the alcohol form versus esterified forms $[5,6]$. Results from these studies have been somewhat variable depending on which ester form has been used. In these studies, the alcohol form shows the highest efficacy as determined using measures such as degrees of sunburn, blistering and skin discolouration. However, the acetate form has shown little or no benefit using the same measures, but surprisingly some benefits have been noted for tocopheryl succinate.

In order to try to understand the observed differences in the effectiveness of the acetate and succinate forms, skin samples treated with each of these two forms analysed following irradiation have demonstrated some liberation of the bioactive free alcohol from the skin samples treated with tocopheryl succinate, but very little free alcohol in the skin treated with the acetate [6]. Although this data suggests potential benefits from the use of the succinate derivative compared with the acetate, the mechanism by which the free alcohol may be liberated from the succinate in skin is still not known.

\section{Ingestion versus topical application}

All the above studies have concentrated on topical application of tocopherols and tocopherol derivatives, but as discussed previously, tocopherols can be secreted into skin layers from dietary depots via the sebaceous glands. The relative benefits of tocopherols supplied by these different routes have been investigated in recent animal studies [7]

In these studies, reductions in skin blistering and pigmentation via topical application of the free alcohol and the succinate forms have been compared with reductions from oral consumption of the acetate form. All three treatments demonstrated benefits in terms of reduced blistering, although the alcohol form was the most effective. Each treatment also showed positive benefits in relation to levels of pigmentation, but in this latter case, similar amounts of benefit were seen for both topical application of the alcohol form and oral intake of the acetate form. In the same study conducted by the Department of Medicine at the Carbrini Medical Centre in New York demonstrated that the topical application of succinate and the consumption of the acetate were seen to reduce the number of tumours when skin is subjected to long-term exposure to UVB radiation [7]. It also revealed a delay in the onset of tumour incidence. This research indicates that oral consumption of vitamin $\mathrm{E}$ may protect the skin from UVB damage, offering additional means for safeguarding it against harmful radiation.

Editor's note: Archer Daniels Midland Company (ADM) is a world leader in agricultural processing. The Company is one of the world's largest processors of soybeans, corn, wheat and cocoa. ADM is also a leader in soy meal and oil, ethanol, corn sweeteners and flour. In addition, ADM is building a position in such valueadded products as specialty food ingredients, bioproducts and nutraceuticals (such as Vitamin $\mathrm{E}$ and sterols). Headquartered in Decatur, Illinois, ADM has over 26000 employees, more than 270 processing plants and net sales for the fiscal year ended June 30, 2003 of $\$ 30.7$ billion. Additional information can be found on ADM's Web site at http://www.admworld.com.

For further product information, please contact: ADM Natural Health and Nutrition, Church Manorway, Erith, Kent, DA8 1DL, UK. Tel: +44 (0)1322 443008 Fax: +44 (0)1322 437536

E-mail: IngredientsErith@admworld.com

\section{REFERENCES}

1. www.bbc.co.uk/health/conditions/skin_cancer (March 2004).

2. THIELE $\|$, TRABER MG, PACKER L. Depletion of human stratum corneum vitamin $E$ : an early and sensitive in vivo marker of UV induced photo-oxidation. I Invest Dermatol $1998 ; 110$ : 756-61.

3. THIELE J], TRABER MG, PACKER L. Sebaceous gland secretion is a major physiologic route of vitamin E delivery to skin. I Invest Dermatol $1999 ; 113: 1006-10$

4. FUCHS I, WEBER S, PODDA M, et al. HPLC analysis of vitamin $E$ isoforms in human epodermis : correlation with minimal erythema dose and free radical scavenging activity. Free Radic Biol Med 2003; 34 : 330-6.

5. MCVEAN M, LIEBLER DC. Prevention of DNA photodamage by vitamin $E$ compounds and sunscreens : roles of ultraviolet absorbance and cellular uptake. 1999.

6. GENSLER HL, AICKIN M, PENG YM, XU M. Importance of the form of topical vitamin $\mathrm{E}$ for prevention of photocarcinogenesis. Nutr Cancer 1996 ; 26 : 183-91.

7. BURKE KE, CLIVE I, COMBS JR. GF, COMMISSO J, KEEN CL, NAKAMURA RM. Effects of topical and oral vitamin $\mathrm{E}$ on pigmentation and skin cancer induced $b$ ultraviolet irradiation in Skh :2 hairless mice. Nutr Cancer 2000; 31 : 87-97. 